\title{
Multitaper Spectrum Sensing of OFDMA Signals in Frequency Selective Fading Environment
}

\author{
Yan Xin, Kyungtae Kim, and Sampath Rangarajan \\ NEC Laboratories America, Inc., Princeton, NJ 08540, USA \\ E-mail: $\{$ yanxin, kyungtae, sampath\}@nec-labs.com
}

\begin{abstract}
This paper considers the problem of how to quickly and accurately identify spectrum holes from downlink orthogonal frequency division multiple access (OFDMA) signals in frequency selective fading environment. We assume that the subcarrier assignment of primary users (PUs) in an OFDMA system is a priori known to the detector. Under this assumption, we formulate the original problem as the problem of detecting presence/absence of PUs, which requires less computational complexity than its original counterpart. We propose a spectrum sensing algorithm to detect presence/absence of a PU. In the proposed algorithm, we first apply the Thomson's multitaper spectrum estimation (MTSE) method to obtain spectral estimates at certain subcarriers of interest, and then we perform a simple threshold test. We present closed form results for false-alarm and miss-detection probabilities of the proposed algorithm. We study impacts of system/MTSE parameters on the detection performance via Monte Carlo simulation.
\end{abstract}

\section{INTRODUCTION}

A major challenge for cognitive radio (CR) is to develop efficient spectrum sensing algorithms that are able to quickly and accurately identify spectrum holes in various wireless environments [1] [2]. In recent years, there has been a significant amount of research effort devoted to such development. Loosely speaking, spectrum sensing techniques can be classified into two categories: narrow-band (single-band) sensing and wide-band (multi-band) sensing.

Although most previous works have focused on narrowband (single-band) spectrum sensing, wide-band (multi-band) spectrum sensing has recently received increasing research attention [3] [4] [5] [6]. More specifically, the work [3] applies a single detector to sequentially detect candidate channels while in [4], multiple sensors with one per candidate channel are employed to simultaneously observe candidate channels. However, when the number of the channels is large, the former suffers from large switching delays introduced by frequently altering observing central frequencies, while the latter has high implementation/operation complexities. Moreover, based on the Thomson's multitaper spectral estimation (MTSE) method, an optimal wide-band detector is proposed in [5] and a wavelet-threshold wide-band sensing technique is proposed in [6]. However, neither of these works considers orthogonal frequency division multiple access (OFDMA) signaling and the effects of frequency selectivity on the detection of primary signals.

In this paper, we consider the problem of how to quickly and accurately identify spectrum holes from downlink OFDMA signals in frequency selective fading environment. We assume that the detector has a priori knowledge on the subcarrier assignment of primary users (PUs) in the system. In an OFDMA system, a set of subcarriers is allocated to a PU and user activities at this set of subcarriers are strongly correlated. The original sensing problem boils down to a problem of detecting presence/absence of PUs. When the number of subcarriers assigned to a PU is large, the new problem requires less computational complexity than its original counterpart because sensing a small part of these subcarriers may be sufficient to achieve a desirable detection performance. To detect presence/absence of a PU, we propose a spectrum sensing algorithm. In the proposed algorithm, we first compute multitaper spectrum estimates at certain subcarriers of interest, and then we perform a simple threshold test. We present closed form results for false alarm and miss detection probabilities of the proposed algorithm. We study impacts of different system/MTSE parameters on the detection performance via Monte Carlo simulation.

\section{System Model And Problem Formulation}

Consider a downlink OFDMA-based primary system that supports maximum $N_{p}$ PUs (indexed by $m \in\left\{1, \ldots, N_{p}\right\}$ ), which share $N_{c}$ sub-carriers (indexed by $k \in\left\{1, \ldots, N_{c}\right\}$ ). The $N_{c}$ sub-carriers are divided to a number of resource blocks (RBs), each of which has $N_{s}$ contiguous sub-carriers. Let $\mathcal{A}$ be the index set of active users with $|\mathcal{A}|$ denoting its cardinality. Without loss of generality, we assume that the $m$ th user is an active user. The information symbol stream of the $m$ th PU is parsed into blocks, each containing $Q$ symbols. Let $\boldsymbol{S}_{m}^{(n)}$ denote the $n$th $Q \times 1$ such block of the $m$ th user. The $q$ th information symbol from this block is denoted by $S_{m, q}^{(n)}$, i.e., $\boldsymbol{S}_{m}^{(n)}=\left[S_{m, 1}^{(n)}, S_{m, 2}^{(n)}, \ldots, S_{m, Q}^{(n)}\right]$. We model primary signal samples $S_{m, q}^{(n)}$ as independent and identically distributed (i.i.d.) random variables (RVs) with means zero and variances $\sigma_{m}^{2}$.

Via serial-to-parallel conversion and subcarrier mapping, information symbols from $|\mathcal{A}|$ active users are fed as inputs to corresponding subcarriers of an $N_{c}$-point inverse fast Fourier transform (IFFT) processor. We use $\kappa_{m, q}$ to denote the subcarrier index of the $q$ th symbol of the $m$ th PU. Let $\boldsymbol{X}^{(n)}$ be the input vector to the IFFT, whose $k$ th entry $X_{k}^{(n)}$ represents the information symbol at the $k$ th subcarrier of the IFFT. Specifically, we can express $X_{k}^{(n)}$ as

$$
X_{k}^{(n)}= \begin{cases}S_{m, q}^{(n)} & \text { if } k=\kappa_{m, q} \text { for } m \in \mathcal{A} \text { and } q \in \mathcal{Q} \\ 0 & \text { otherwise }\end{cases}
$$


where $\mathcal{Q}:=\{1, \ldots, Q\}$. The output vector of the IFFT is obtained as $\boldsymbol{x}^{(n)}=F_{N_{c}}^{H} \boldsymbol{X}^{(n)}$, where $F_{N_{c}}^{H}$ denotes the conjugate transpose of the normalized FFT matrix of size $N_{c}$. After parallel-to-serial conversion, a cyclic prefix of length $N_{g}$ is appended in front of $\boldsymbol{x}^{(n)}$. The $i$ th entry of the resulting vector is given by $x_{i}^{(n)}=N_{c}^{-\frac{1}{2}} \sum_{k=1}^{N_{c}} X_{k}^{(n)} e^{j 2 \pi i k / N_{c}}$, if $N_{g}+1 \leq i \leq N_{c}$ and $x_{i}^{(n)}=0$, otherwise. Let $x_{s}$ be the serialized version of the blocks $\boldsymbol{x}^{(n)}$ and let $N_{u}$ be the length of a transmission block, i.e., $N_{u}=N_{g}+N_{c}$. Specifically, $x_{s}$ is defined as follows $x_{s}:=x_{i}^{(n)}$, where $s:=n N_{u}+i$. Here we assume that the channel between the primary transmitter and the detector at the secondary user (SU) is time-invariant and frequency selective. Let $\boldsymbol{h}=\left[h_{0}, \ldots, h_{L}\right]$ denote the sampled impulse response of the channel, where $L$ is the channel length and $h_{l}$ is the $l$ th channel tap. The $t$ th received signal sample at the detector is given as

$$
r_{t}=e^{j 2 \pi \delta t / N_{c}} \sum_{l=0}^{L} h_{l} x_{t-l}+w_{t}
$$

where $\delta$ denotes a normalized frequency offset, and $w_{t}$ is additive white Gaussian noise (AWGN) with variance $\sigma_{w}^{2}$. Here, we assume that 1) the primary signal $x_{t}$ is independent of $\left.w_{t} ; 2\right) \delta$ is much smaller than the subcarrier spacing; 3 ) the channel impulse response $\boldsymbol{h}$ is perfectly known to the detector; and 4) the SU has a priori knowledge on primary system specifications, such as the central frequency, the sampling period, the subcarrier spacing and assignment, and etc.

Remark 1: Assumption 3) is made primarily for deriving an exact error performance of our detection method, which will be presented in Section IV. For certain cellular systems based on 3rd generation partnership project (3GPP) long term evolution (LTE) or IEEE 802.16 standards, system specifications such as the subcarrier assignment, preamble, and frame structure, are publicly available. Assumption 4) can be satisfied by exploiting such information.

During a given time period, certain PUs are not active and thus the spectrum bands allocated to these PUs become available for secondary use. Due to the assumption that users' activities are independent, we only need to focus on a single active PU. Without loss of generality, we consider the $m$ th PU. Mathematically, the problem of detecting the $m$ th PU can be formulated as a binary hypothesis testing problem as

$$
\begin{aligned}
& H_{0}: X_{\kappa_{m}, q}^{(n)}=0, \quad q \in \mathcal{Q} \\
& H_{1}: X_{\kappa_{m, q}}^{(n)}=S_{m, q}^{(n)}, \quad q \in \mathcal{Q} .
\end{aligned}
$$

\section{Multitaper Spectral Estimation}

We next review the Thomson's MTSE method and some necessary statistical properties of MTSE [7].

\section{A. Preliminary}

The MTSE method employs several orthogonal tapers (windows). Let $\left\{a_{t, p}\right\}_{t=1}^{N}, p=1, \ldots, P$, be $P$ orthogonal tapers of length $N$, which satisfy $\sum_{t=1}^{N} a_{t, l} a_{t, p}=1$ if $l=p$ and $\sum_{t=1}^{N} a_{t, l} a_{t, p}=0$ if $l \neq p$. In practice, two families of orthogonal tapers, called the Slepian tapers [7] and the sinusoidal tapers [8], are commonly used. Here we assume that the sampling interval is unity. In particular, the sinusoidal taper is given in an analytical form as

$$
a_{t, p}=\sqrt{\frac{2}{N+1}} \sin \left(\frac{\pi p t}{N+1}\right)
$$

where $t=1, \ldots, N$ and $p=1, \ldots, P$. For a stationary discrete time series $\left\{r_{t}\right\}_{t=1}^{N}$, the $p$ th taper spectral estimator (eigenspectra) is given by

$$
\hat{R}_{p}(f)=\left|\sum_{t=1}^{N} a_{t, p} r_{t} e^{-j 2 \pi t f}\right|^{2},|f|<\frac{1}{2} .
$$

The multitaper spectral estimate is the average of $P$ taper spectrum estimates and is given by $\hat{R}(f)=\sum_{p=1}^{\mathcal{P}} \hat{R}_{p}(f) / P$. Let $2 W$ be the resolution bandwidth. The parameter $P$ is often selected to be $2 N W-1$ for Slepian tapers and $2 W(N+1)-1$ for sinusoidal tapers.

\section{B. Statistical Properties of MTSE}

We adopt the assumption in [9] that the spectrum varies slowly over $(f-W, f+W)$. Let $R(f)$ denote the true power spectral density (PSD) function of $r_{t}$.

1) Mean, Variance, and Convergence: For a large $N$, the mean and variance of $\hat{R}(f)$ can be approximated as $E[\hat{R}(f)] \approx R(f)$, and $\operatorname{var}[\hat{R}(f)] \approx R^{2}(f) / P$, and $\hat{R}(f)$ converges in distribution to $R(f) \chi_{2 P}^{2} /(2 P)$ as $N$ approaches $\infty$ for $f \in(0,1 / 2)$ [9].

2) Covariance of Multitaper Spectrum Estimates at Two Distinct Frequencies: We now further assume that the time series $r_{t}$ is a realization of a Gaussian process with mean zero. Let $f_{i}$ and $f_{j}$ be two distinct frequencies. If $R(f)$ is slow varying over $\left(f_{i}-W, f_{j}+W\right)$, the covariance of $\hat{R}\left(f_{i}\right)$ and $\hat{R}\left(f_{j}\right)$ can be written as [9]

$$
\Gamma_{i j} \approx \begin{cases}\frac{R^{2}\left(f_{i}\right)}{P^{2}} \sum_{l, p=1}^{P} \Lambda_{l, p}\left(\Delta_{i j}\right) & \text { if }\left|\Delta_{i j}\right| \leq 2 W \\ 0 & \text { if }\left|\Delta_{i j}\right|>2 W\end{cases}
$$

where $\Gamma_{i j}:=\operatorname{cov}\left[\hat{R}\left(f_{i}\right), \hat{R}\left(f_{j}\right)\right], \Delta_{i j}:=f_{i}-f_{j}$ and $\Lambda_{l, p}\left(\Delta_{i j}\right):=\left|\sum_{t=1}^{N} a_{t, l} a_{t, p} e^{j 2 \pi \Delta_{i j} t}\right|^{2}$.

\section{The MtSE Based Detection Method}

To detect the presence/absence of a PU, we propose a Multitaper spectrum estimation based Threshold Test (MTT) as follows:

$$
\mathcal{T}:=\sum_{u=1}^{N_{f}} \hat{R}\left(f_{u}\right) \underset{H_{0}}{\stackrel{H_{1}}{\gtrless}} \eta_{t h}
$$

where $\left\{f_{u}\right\}_{u=1}^{N_{f}}$ denotes a set of sampling frequencies from the allocated spectrum of a PU, $N_{f}$ denotes the number of the sampling frequencies, and $\eta_{t h}$ denotes a threshold. Without loss of generality, we assume that the frequencies $\left\{f_{u}\right\}_{u=1}^{N_{f}}$ are equally spaced, and the frequency separation is denoted by $\Delta$, i.e., $\Delta:=\left|f_{u+1}-f_{u}\right|$. 
Like energy detection, the threshold test in (4) has a simple test statistic and performs a threshold comparison to determine hypothesis $H_{0}$ or $H_{1}$. There are, however, some important differences between energy detection and MTT. First, the terms in the test statistic of energy detection is often assumed to be i.i.d. and the number of the terms is typically large. As a result, the central limit theorem (CLT) can be applied to approximate the probability density function (PDF) of the test statistic. In the threshold test (4), the terms $\left\{\hat{R}\left(f_{u}\right)\right\}_{u=1}^{N_{f}}$ are not necessarily independent and the number of these terms is not necessarily large. Thus, CLT is no longer applicable. The PDF of the test statistic in (4) is much more complicated than that in energy detection. Secondly, for a given signal-tonoise ratio (SNR), the detection error performance of energy detection is determined by the threshold and the number of the terms in the test statistic. Besides these two parameters $\eta_{t h}$ and $N_{f}$ in (4), the detection error performance of MTT is also determined by the number of samples, $N$, the number of tapers, $P$, and the frequency separation, $\Delta$. As discussed earlier, $\left\{\hat{R}\left(f_{u}\right)\right\}_{u=1}^{N_{f}}$ are Chi-square distributed for a large $N$ and they are not necessarily independent. Statistically, the test statistic $\mathcal{T}$ is nothing but a sum of the correlated Chi-square RVs. We next will present results on the PDF of $\mathcal{T}$ in [10].

\section{A. The Sum of Correlated Chi-Square Random Variables}

We use $\gamma_{u}$ as a shorthand notation to represent $\hat{R}\left(f_{u}\right)$. Let $\boldsymbol{R}$ denote the $N_{f} \times N_{f}$ covariance matrix of the vector $\left[\gamma_{1}, \ldots, \gamma_{N_{f}}\right]$ with $r_{i j}$ denoting its $(i, j)$ th entry. Let $\boldsymbol{C}$ be a matrix that relates to $\boldsymbol{R}$ as $c_{i j}=\sqrt{r_{i j} / P}, i, j=1, \ldots, N_{f}$, where $c_{i j}$ is its $(i, j)$ th entry. Let $\left\{\lambda_{l}\right\}_{l=1}^{N_{e}}$ be $N_{e}$ distinct eigenvalues of $C$ and let $\left\{m_{l}\right\}_{l=1}^{N_{e}}$ be their corresponding multiplies. Note that each $\gamma_{s}$ has the same $P$. Extending the result in [10, Eq. (20)], we obtain the PDF of $\mathcal{T}$ as $f(\mathcal{T})=\sum_{l=1}^{N_{e}} \sum_{r=1}^{P m_{l}} \beta_{l r} p(\mathcal{T} ; l, r)$, where $\beta_{l r}$ is defined as

$$
\begin{aligned}
& \text { for } r=P m_{l}, \beta_{l r}=\left.\left(\prod_{k=1, k \neq l}^{N_{e}}\left(1-s \lambda_{k}\right)^{-P m_{k}}\right)\right|_{s=1 / \lambda_{l}}, \\
& \text { for } 0<r<P m_{l}, \quad \beta_{l r}=\frac{1}{\left(P m_{l}-1\right) !\left(-\lambda_{l}\right)^{P m_{l}-r}} \\
& \qquad\left.\frac{d^{P m_{l}-r}}{d s^{P m_{l}-r}} \prod_{k=1, k \neq l}^{N_{e}}\left(1-s \lambda_{k}\right)^{-P m_{l}}\right|_{s=1 / \lambda_{l}}
\end{aligned}
$$

and $p(\mathcal{T} ; l, r)$ is defined as

$$
p(\mathcal{T} ; l, r)=\frac{1}{\lambda_{l}(r-1) !}\left(\mathcal{T} / \lambda_{l}\right)^{r-1} e^{-\mathcal{T} / \lambda_{l}} .
$$

In particular, when $\left\{\gamma_{u}\right\}_{u=1}^{N_{f}}$ are i.i.d., we have $N_{e}=1$ and $m_{1}=N_{f}$. Accordingly, the PDF of $\mathcal{T}$ reduces to

$$
f(\mathcal{T})=\frac{1}{\lambda_{1}\left(N_{f} P-1\right) !}\left(\mathcal{T} / \lambda_{1}\right)^{N_{f} P-1} e^{-\mathcal{T} / \lambda_{1}} .
$$

Note that in this case, we have $\operatorname{var}\left[r_{i i}\right]=R^{2}\left(f_{i}\right) / P$. Since $\boldsymbol{R}$ is a diagonal matrix with the diagonal entries $\operatorname{var}\left[r_{i i}\right], C$ is also a diagonal matrix with the diagonal entries $R\left(f_{i}\right) / P$ as $c_{i i}=\sqrt{r_{i i} / P}$.

\section{B. The False-Alarm Probability}

Under $H_{0}$, the detector observes AWGN $w_{t}$. Hence, the true PSD $R(f)$ is constant and is equal to $\sigma_{w}^{2}$. According to (3) and the fact that $c_{i j}=\sqrt{r_{i j} / P}$, we obtain $c_{i j}$ as

$$
c_{i j}= \begin{cases}\frac{\sigma_{w}^{2}}{P} \sqrt{\frac{\sum_{l, p=1}^{N} \Lambda_{l, p}\left(D_{i j}\right)}{P}}, & \text { if } D_{i j} \leq 2 W \\ 0, & \text { if } D_{i j}>2 W\end{cases}
$$

where $D_{i j}:=|i-j| \Delta$. The false-alarm probability is defined as the probability of the event that a PU is determined to be active while actually the PU is inactive. Mathematically, the false alarm probability is given by $P_{F A}=P\left(\mathcal{T}>\eta_{t h} \mid H_{0}\right)=$ $\int_{\eta_{t h}}^{\infty} f\left(\mathcal{T} \mid H_{0}\right) d \mathcal{T}$. We next present the following result on the false-alarm probability $P_{F A}$.

Proposition 1: The false alarm probability $P_{F A}$ is given by

$$
P_{F A}=\sum_{l=1}^{N_{e}} \sum_{r=1}^{P m_{l}} \beta_{l r} \frac{\Gamma\left(r, \eta_{t h} / \lambda_{l}\right)}{(r-1) !}
$$

where $\Gamma(\cdot, \cdot)$ denotes the upper incomplete Gamma function, i.e., $\Gamma(s, x)$ is defined as $\Gamma(s, x)=\int_{x}^{\infty} t^{s-1} e^{-t} d t$, and $N_{e}$, $m_{l}$ and $\lambda_{l}$ are the corresponding parameters for the matrix $C$ given in (5). In particular, if $\left\{\hat{R}\left(f_{u}\right)\right\}_{u=1}^{N_{f}}$ are i.i.d., then $P_{F A}$ is given by

$$
P_{F A}=\frac{\Gamma\left(N_{f} P, \eta_{t h} / \lambda_{1}\right)}{\left(N_{f} P-1\right) !}
$$

and for given $N_{f}, P$ and $P_{F A}$, the threshold can be determined as $\eta_{t h}=\Gamma^{-1}\left(N_{f} P, P_{F A}\left(N_{f} P-1\right) !\right) \lambda_{1}$, where $\Gamma^{-1}$ denotes the inverse of the incomplete Gamma function $\Gamma(s, x)$ with respect to the second parameter $x$.

\section{The Miss-Detection Probability}

We now compute the miss-detection probability. Under $H_{1}$, the received signal samples in (1) can be rewritten as $r_{t}=\sum_{l=0}^{L} h_{l} u_{t-l}+w_{t}$, where $u_{t}:=x_{t} e^{j 2 \pi \delta t / N_{c}}$. Recall that $S_{m, 1}^{(n)}, \ldots, S_{m, Q}^{(n)}$ are i.i.d. RVs. Since $Q$ is relatively large in practice, the transmitted signal samples $x_{t}$ can be approximately modelled by a Gaussian random process. Since a linear transform of a Gaussian process is still a Gaussian process, the received samples $r_{t}$ can be also approximated treated as a realization of a Gaussian process. Hence, the results on the covariance of the MTSE between two distinct frequencies in Section III hold. According to (3), we need to obtain the true PSD, $R(f)$, in order to compute an approximation of $c_{i j}$, This, however, is infeasible in practice. Here, we use the unbiased property of MTSE, i.e., $E[\hat{R}(f)]=R(f)$, to reexpress $c_{i j}$ as

$$
c_{i j}= \begin{cases}\frac{E[\hat{R}(f)]}{P} \sqrt{\frac{\sum_{l, p}^{N} \Lambda_{l, p}\left(D_{i j}\right)}{P}}, & \text { if } D_{i j} \leq 2 W \\ 0, & \text { if } D_{i j}>2 W .\end{cases}
$$

We express the $p$ th taper spectral estimate under $H_{1}$ as $\hat{R}_{p}(f)=\left|\sum_{l=0}^{L} h_{l} X_{p, l}(f)+W_{p}(f)\right|^{2}$, where $X_{p, l}(f):=$ $\sum_{t=1}^{N} a_{t, p} u_{t-l} e^{-j 2 \pi t f}$ and $W_{p}(f):=\sum_{t=1}^{N} a_{t, p} w_{t} e^{-j 2 \pi t f}$. 
Using the facts that $X_{p, l}(f)$ and $W_{p}(f)$ are independent and $E_{1}\left[\left|W_{p}(f)\right|^{2}\right]=\sigma_{w}^{2}$, we can write $E_{1}\left[\hat{R}_{p}(f)\right]$ as

$$
E_{1}\left[\hat{R}_{p}(f)\right]=E_{1}\left[\left|\sum_{l=0}^{L} h_{l} X_{p, l}(f)\right|^{2}\right]+\sigma_{w}^{2},
$$

where $E_{1}[\cdot]$ denotes the conditional expectation under $H_{1}$. Furthermore, we have

$$
E_{1}\left[\left|\sum_{l=0}^{L} h_{l} X_{p, l}(f)\right|^{2}\right]=\sum_{l_{1}, l_{2}=0}^{L} h_{l_{1}} h_{l_{2}}^{*} E_{1}\left[X_{p, l_{1}}(f) X_{p, l_{2}}^{*}(f)\right] .
$$

Let $\sigma_{k}^{2}$ be the transmitted signal power at subcarrier $k$, i.e., $\sigma_{k}^{2}=\sigma_{m}^{2}$ if $k=\kappa_{m, q}$ for $m \in \mathcal{A}$ and $q \in \mathcal{Q}$ and $\sigma_{k}^{2}=0$ otherwise. Under certain assumptions, we obtain the following results on $E_{1}\left[\left|\sum_{l=0}^{L} h_{l} X_{p, l}(f)\right|^{2}\right]$.

Proposition 2: If $N$ is sufficiently large and a multiple of $N_{c}$, i.e., $N=N_{l} N_{c}$ for a positive integer $N_{l}$, then

$$
\begin{aligned}
E_{1}\left[\left|\sum_{l=0}^{L} h_{l} X_{p, l}(f)\right|^{2}\right]= & \sum_{l_{1}, l_{2}=0}^{L} h_{l_{1}} h_{l_{2}}^{*} \sum_{k=1}^{N_{c}} \sigma_{k}^{2} e^{j \frac{2 \pi k\left(l_{1}-l_{2}\right)}{N_{c}}} \\
& \sum_{s=0}^{N_{l}-1} A_{k, s}(f ; p)
\end{aligned}
$$

where $A_{k, s}(f ; p)=\left|\sum_{i=1}^{N_{c}} a_{s N_{c}+i, p} e^{-j 2 \pi \zeta_{k} i}\right|^{2}$ with $\zeta_{k}:=f-$ $k / N_{c}$.

Lemma 1: For sinusoidal tapers, $A_{k, s}(f ; p)$ is given by

$$
\begin{aligned}
A_{k, s}(f ; p)=\frac{1}{2(N+1)} \mid & \Xi_{s, p} \frac{\sin \left(\pi\left(V_{p} / 2-\zeta_{k}\right) N_{c}\right)}{\sin \left(\pi\left(V_{p} / 2-\zeta_{k}\right)\right)} \\
& -\left.\Xi_{s, p}^{*} \frac{\sin \left(\pi\left(V_{p} / 2+\zeta_{k}\right) N_{c}\right)}{\sin \left(\pi\left(V_{p} / 2+\zeta_{k}\right)\right)}\right|^{2}
\end{aligned}
$$

where $\Xi_{s, p}:=e^{j \pi U_{s, p}} e^{j \pi V_{p}\left(N_{c}+1\right) / 2}$ with $U_{s, p}:=p s N_{c} /(N+$ 1) and $V_{p}:=p /(N+1)$.

Corollary 1: For sinusoidal tapers, if $N_{c}$ is less than or equal to $1 / 2 W$, and $N$ is a multiple of $N_{c}$ and is much larger than $N_{c}$, i.e., $N_{l} \gg 1$, then

$$
\begin{aligned}
E_{1}\left[\left|\sum_{l=0}^{L} h_{l} X_{p, l}(f)\right|^{2}\right] \approx & \frac{N_{l}}{N+1} \sum_{l_{1}, l_{2}=0}^{L} h_{l_{1}} h_{l_{2}}^{*} \sum_{k=1}^{N_{c}} \sigma_{k}^{2} \\
& e^{j 2 \pi k \frac{l_{1}-l_{2}}{N_{c}}}\left|\frac{\sin \left(\pi \zeta_{k} N_{c}\right)}{\sin \left(\pi \zeta_{k}\right)}\right|^{2} .
\end{aligned}
$$

In particular, if $f=k_{0} / N_{c}$ for some $k_{0} \in\left\{1, \ldots, N_{c}\right\}$,

$$
E_{1}\left[\left|\sum_{l=0}^{L} h_{l} X_{p, l}\left(\frac{k_{0}}{N_{c}}\right)\right|^{2}\right] \approx \sigma_{k_{0}}^{2}\left|H\left(\frac{k_{0}}{N_{c}}\right)\right|^{2}
$$

where $H(f):=\sum_{l=0}^{L} h_{l} e^{j 2 \pi l f}$.

Remark 2: Lemma 1 and Corollary 1 hold only for sinusoidal tapers. For the Slepian tapers, it is difficult to obtain an analytical form of $A_{k, s}(f ; p)$ and an analytical expression of $E_{1}\left(\left|\sum_{l=0}^{L} h_{l} X_{p, l}(f)\right|^{2}\right)$. However, via simulation, a similar result to the one in (10) can be observed.
Applying Corollary 1 , we have $E_{1}\left(\hat{R}_{p}\left(i / N_{c}\right)\right) \approx$ $\sigma_{i}^{2}\left|H\left(i / N_{c}\right)\right|^{2}+\sigma_{w}^{2}$, which is independent of the taper in$\operatorname{dex} p$ for any $i \in\left\{1, \ldots, N_{c}\right\}$. Since $E_{1}\left(\hat{R}\left(i / N_{c}\right)\right)=$ $E_{1}\left(\hat{R}_{p}\left(i / N_{c}\right)\right)$ and $E_{1}\left(\hat{R}\left(i / N_{c}\right)\right) \approx R\left(i / N_{c}\right)$, we have $R\left(i / N_{c}\right) \approx \sigma_{k_{0}}^{2}\left|H\left(i / N_{c}\right)\right|^{2}+\sigma_{w}^{2}$. In practice, the frequency response $H\left(i / N_{c}\right)$ can be approximated as a constant over a $\mathrm{RB}$, which is much larger than $2 W$. Thus, the diagonal entries $c_{i i}$ are given by $c_{i i}=\left(\sigma_{i}^{2}\left|H\left(i / N_{c}\right)\right|^{2}+\sigma_{w}^{2}\right) / P$.

The miss detection probability is defined as the probability of the event that a PU is determined to be inactive while actually the PU is active. Mathematically, the miss detection probability is given by

$$
P_{M D}=P\left(T<\eta_{t h} \mid H_{1}\right)=\int_{0}^{\eta_{t h}} f\left(\mathcal{T} \mid H_{1}\right) d \mathcal{T} .
$$

Proposition 3: The miss-detection probability $P_{M D}$ is given by

$$
P_{M D}=\sum_{l=1}^{N_{e}} \sum_{r=1}^{P m_{l}} \beta_{l r} \frac{\gamma\left(r, \frac{\eta_{t h}}{\lambda_{l}}\right)}{(r-1) !}
$$

where $\gamma(\cdot, \cdot)$ denotes the lower incomplete gamma function, i.e., $\gamma(s, x)$ is defined as $\gamma(s, x)=\int_{0}^{x} t^{s-1} e^{-t} d t$, and $N_{e}, m_{l}$ and $\lambda_{l}$ are the corresponding parameters for $C$ in (8).

Remark 3: For given $P_{F A}$ and $P_{M D}$, the parameters $N$, $N_{f}, P$, and $\eta_{t h}$ can be obtained by using a trial and error method in [11] (omitted due to limited space), which relies heavily on Propositions 1 and 3.

\section{Simulation Results}

We next provide several simulation examples to demonstrate the effectiveness of the MTT method. In all the examples, we choose $N_{s}=N_{f}$ and $\Delta=1 / N_{c}$, and we use Slepian tapers unless explicitly mentioned. We assume that $E\left(\left|S_{m, q}^{(n)}\right|^{2}\right)=$ $\sigma_{s}^{2}$ independent of $q$. The SNR is defined as $\sigma_{s}^{2} / \sigma_{w}^{2}$. We use operating characteristic curves as our performance benchmark.

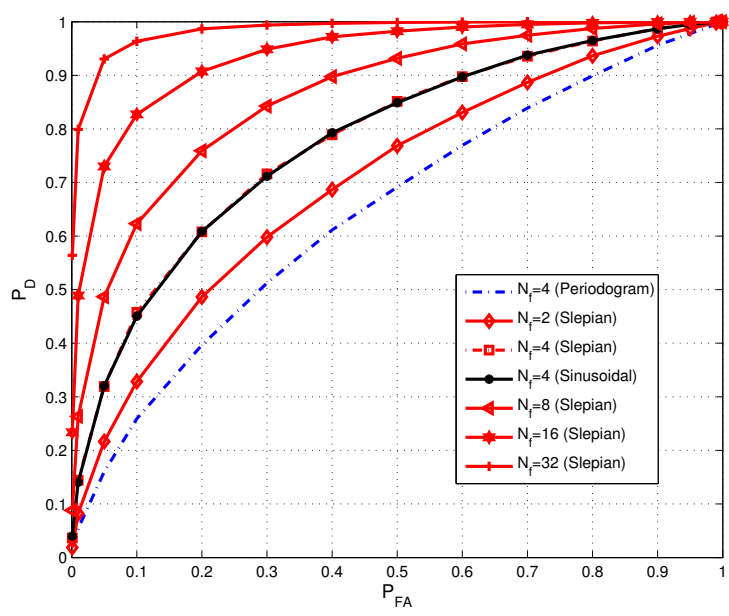

Fig. 1. Impacts of the number of sampling points $N_{f}$ and different tapers $\left(N_{c}=64, N=2048, P=4, \mathrm{SNR}=-5 \mathrm{~dB}\right)$. 
TABLE I

Comparisons of Simulation AND NUMERiCAl Results $(N=2048$, $N_{c}=256, P=4, N_{f}=8$ )

\begin{tabular}{c|c|c|c|c|c|c|c}
\hline$\eta_{t h}$ & 9.16 & 8.68 & 8.28 & 7.92 & 7.57 & 7.21 & 6.79 \\
\hline \hline$P_{F A}(N)$ & 0.20 & 0.30 & 0.40 & 0.50 & 0.60 & 0.70 & 0.80 \\
\hline$P_{F A}(S)$ & 0.20 & 0.31 & 0.40 & 0.51 & 0.60 & 0.71 & 0.80 \\
\hline \hline$P_{M D}(N)$ & 0.29 & 0.20 & 0.14 & 0.092 & 0.059 & 0.035 & 0.018 \\
\hline$P_{M D}(S)$ & 0.30 & 0.21 & 0.14 & 0.096 & 0.060 & 0.037 & 0.018 \\
\hline
\end{tabular}

Example 1: In this example, we consider an AWGN channel with $h_{0}=1$. We choose $N_{c}=64, N=2048, P=4$, and $\mathrm{SNR}=-5 \mathrm{~dB}$. In this case, the frequency separation $\Delta$ is $6.4 \mathrm{~W}$ much larger than $2 \mathrm{~W}$, and thus multitaper spectral estimates $\left\{\gamma_{u}\right\}_{u=1}^{N_{f}}$ can be considered to be independent. Fig. 1 depicts operating characteristic curves of the MTT using Slepian tapers for various values of $N_{f}$. As can be seen from this figure, the detection performance improves as $N_{f}$ increases. Fig. 1 also compares the detection performance among Slepian tapers, sinusoidal tapers, and periodogram (single taper). As shown in the figure, Slepian and Sinusoidal tapers have almost the same performance and both perform much better than the periodogram.

Example 2: Table I lists false-alarm and miss-detection probabilities obtained from (6) and (11) and from the Monte Carlo simulation for $N=2048, N_{c}=256, N_{f}=8$, $P=4$, and $\mathrm{SNR}=-5 \mathrm{~dB}$. We consider a frequency selective channel with $L=3$ with known but randomly generated channel coefficients, where $\boldsymbol{h}=[0.7288-0.5988 i,-0.0520+$ $0.0247 i, 0.081+0.1021 i, 0.0208+0.0038 i]$. In this table, we use $P_{F A}(N)$ and $P_{M D}(N)$ to denote false alarm probability and miss detection probabilities obtained from (6) and (11), respectively, whereas we use $P_{F A}(S)$ and $P_{M D}(S)$ to denote false alarm and miss detection probabilities obtained by using Monte Carlo simulation, respectively. As can be seen from Table I, the simulation results match very well with the numerical results obtained from (6) and (11).

Example 3: In this example, we consider a three tap frequency selective fading channel with an exponentially decaying power-delay profile $(L=3)$. We do not assume perfect knowledge on $\boldsymbol{h}$ and we only assume that the total energy of the channel coefficients is known and is equal to unity, i.e, $\sum_{l=0}^{3}\left|h_{l}\right|^{2}=1$. We choose $N_{c}=128, Q=8, N=2048$, and $\mathrm{SNR}=0 \mathrm{~dB}$. We study impacts of frequency diversity gains on the detection performance. Let $N_{b}$ denote the number of RBs of a PU. We consider two cases: case 1) the PU has two RBs each having 4 contiguous subcarriers, and two RBs are separated by 60 subcarriers, and case 2 ) the PU has only one RB that consists of 8 contiguous subcarriers. Potentially, case 1) has a larger frequency diversity gain than case 2). As shown in Fig. 2, case 1) has a better detection performance than case 2) due to frequency selectivity of the channels.

\section{CONCLUSIONS}

In this paper, we have investigated how to sense OFDMA signals in frequency selective fading environment. Based on

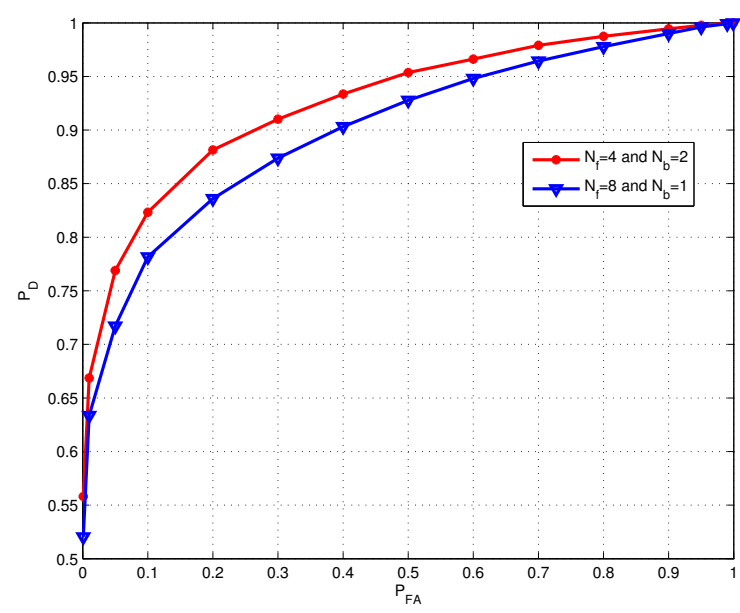

Fig. 2. Impacts of frequency selectivity on detection performance $\left(N_{c}=\right.$ $128, Q=8, N=2048, L=3$, and SNR $=0 \mathrm{~dB}$ ).

the Thomson's MTSE method, we have proposed a simple spectrum sensing algorithm to detect spectrum holes of downlink OFDMA primary systems. We have derived closed form results for false-alarm and miss-detection probabilities of the proposed algorithm. We have investigated impacts of various parameters on the detection performance. In this work, impacts of timing and frequency offsets on the detection performance are not taken into account and they are currently under investigation.

\section{REFERENCES}

[1] S. Haykin, D. J. Thomson, and J. H. Reed, "Spectrum sensing for cognitive radio," Proc. IEEE, vol. 97, no. 5, pp. 849-877, May 2009.

[2] Y.-C. Liang, Y. Zeng, E. Peh, and A. Hoang, "Sensing-throughput tradeoff for cognitive radio networks," IEEE Trans. Wireless Commun., vol. 7, no. 4, pp. 1326-1337, Apr. 2008.

[3] Y. Xin and L. Lai, "Fast wideband spectrum scanning for multi-channel cognitive radio systems," in Proc. of the 44th Annual Conference on Information Sciences and Systems, Princeton University, Princeton, NJ, March 17-19, 2010.

[4] S. J. Kim and G. B. Giannakis, "Rate-optimal and reduced-complexity sequential sensing algorithms for cognitive ofdm radios," in Proc. 43rd Conf. on Info. Sciences and Systems, Johns Hopkins Univ., Baltimore, MD, Mar. 18 - 20, 2009.

[5] T. W. Chiang, J. M. Lin, and H. P. Ma, "Optimal detector for multitaper spectrum estimator in cognitive radios," in Proc. IEEE Global Communications Conference (GLOBECOM), Honolulu, HI, USA, Nov. 30-Dec.4, 2009.

[6] J. Tugnait, "Wavelet-thresholded multitaper spectrum sensing for cognitive radios in unknown noise," in Proc. of the 2010 IEEE International Conf. on Communications (ICC), Cape Town, South Africa, May 24-27, 2010.

[7] D. J. Thomson, "Spectrum estimation and harmonic analysis," Proc. IEEE, vol. 70, no. 9, pp. 1055-1096, Sept. 1982.

[8] K. S. Riedel and A. Sidorenko, "Minimum biased multitaper spectral estimation," IEEE Trans. Signal Processing, vol. 43, no. 1, pp. 188195, Jan. 1995.

[9] D. B. Percival and A. T. Walden, Spectral Analysis for Physical Applications: Multitaper and Conventional Univariate Techniques. Cambridge, U.K.: Cambridge University Press, 1993.

[10] Q. T. Zhang, "Maximum-ratio combining over Nakagami fading channels with an arbitrary branch covariance matrix," IEEE Trans. Veh Technol., vol. 48, no. 4, pp. 1141-1150, July 1999.

[11] Y. Xin, K. Kim, and S. Rangarajan, "Multitaper spectrum sensing of ofdma signals in frequency selective fading environment," Technical Report, NEC Laboratories America, Inc., Feb. 2011. 